\title{
Towards a Robust Concurrent and Interleaved Activity Recognition of Mobile Users
}

\author{
Saguna ${ }^{1,2}$, Arkady Zaslavsky ${ }^{1,2}$ \\ ${ }^{1}$ Monash University, Melbourne, Victoria, Australia \\ ${ }^{2}$ Luleå University of Technology, Luleå, Sweden, \\ saguna.saguna@monash.edu, arkady.zaslavsky@ltu.se
}

\author{
Dipanjan Chakraborty \\ IBM Research, India Research Lab, New Delhi, India \\ cdipanjan@in.ibm.com
}

\begin{abstract}
This paper proposes a situation and context-aware complex activity recognition system where we use on-body sensor information along with other context information to infer mobile user activities which are both concurrent and interleaved. We develop and validate our complex activity recognition algorithm for detecting these multiple complex activities. Activities are mapped onto situations using spatio-temporal analysis. We further build a test-bed in the social-networking domain to test and validate our approach in different scenarios using mobile devices.
\end{abstract}

\section{Keywords-activity recognition; context awareness; situations}

\section{INTRODUCTION}

Human activity recognition has witnessed an increased interest from the research community in recent years with its applications in areas such as health monitoring, aged care, industry, sports and military [1]. Humans while performing activities of daily living (ADL) mostly multi-task between these highly complex ADLs with ease as most operations are routinized [2]. ADLs can be personal or professional activities performed at home, office or in the outdoors. The recognition of ADLs has significant potential in terms of developing smarter environments for mobile and ubiquitous computing. A large body of work exists in the field of activity analysis which focuses on atomic activities. For example, a complex activity like "cooking pasta" can have many atomic activities like "picking a pan" and "picking a pasta box". Similarly, "watching television" can have atomic activities like "sitting on couch" and "using a remote control". All human activity recognition problems somewhat follow a common approach. This includes the use of sensors, multi-sensor fusion (datalevel, feature-level or decision-level), the segmentation of data with relation to time and space, feature extraction and selection, classification of activities, and the use of activity models to infer activities [1].

In workshop and sports settings, sequence is of immense importance while performing activities. This is not true for ADLs where sequence is followed rarely and only for a very few activities. For example, users while cooking pasta, may first boil the pasta or cut the vegetables. Such sequences always tend to change. Also, complex ADLs tend to be performed by a user in a concurrent or interleaved manner. There are problem areas such as activity labelling for large datasets and creating models for activities every time there is a change in sequence or new activities are added. Most existing models are sensitive to such changes or additions of new activities. In this paper, we develop a novel situation- and context-aware activity recognition system which is used to infer concurrent and interleaved complex activities. To this extent, we look at activities from the perspective of events, atomic or composite. We then infer situations and link activities to situations. These situations are an extension of the concept of composite events [3] but we differentiate them from complex activities. We only consider situations which are inferred from sensor and context information not linked directly to activities, for example, temperature, light in a room, location. Sensor and context information directly linked to activity would imply sensors like accelerometers and context information like speed from GPS.

\section{MOTIVATING SCENARIO}

Iris is an employee of a company, say future.com. She spends her day doing a number of activities like laundry, cooking, watching television and office work. She has office hours Monday to Friday from 9:00AM to 5:00PM. On a Monday morning, she may be getting ready, preparing her breakfast, watching morning news, reading a research article, etc. Similarly, in office she may be reading documents, creating presentations, attending meetings and drinking coffee. All these are examples of complex activities which comprise of atomic activities that are undertaken concurrently or interleaved amongst each other. Also, she constantly makes presence updates about her different activities to her preferred social interaction tools to be shared with her friends and family

\section{SACAAR ARCHITECTURE}

Our proposed Situation- And Context-Aware Activity Recognition (SACAAR) architecture is shown in figure 1. The sensory layer collects sensor and context information from different wearable sensors, virtual sensors and other sources in the environment such as mobile devices or the web. Wearable sensors include accelerometers for body motion, Bluetooth RFID reader wristband for object interaction, GPS for outdoor location, Ekahau WLAN tag for indoor location, RFID for indoor location within a room and physiological sensors like heart rate monitors for mood as well as stress levels. Virtual sensors can include modules of code which gather device activity, browser activity from a user's devices. Context information includes location, time, calendar entries, light on/off, etc. Data is stored in SACAAR in a distributed environment in multiple locations. The use of mobile devices is crucial for SACAAR in inferring complex activities. The data 


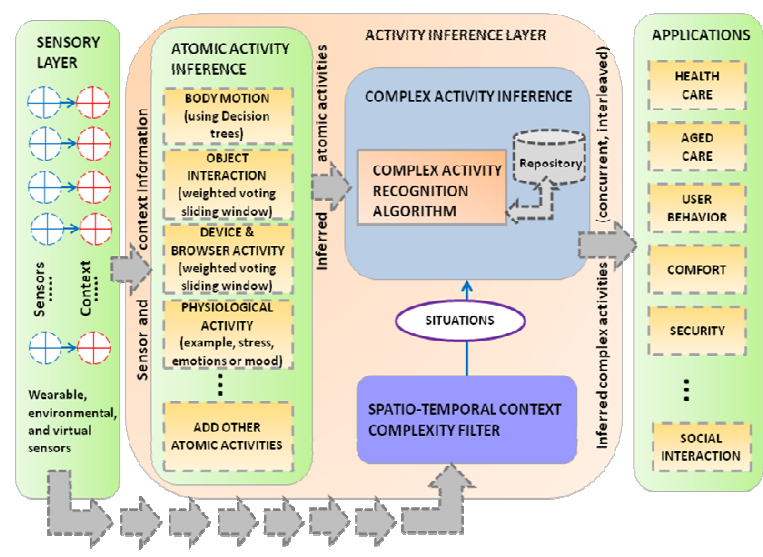

Figure 1. SACAAR architecture.

is constantly exchanged between mobile devices in our distributed environment. Raw data is exchanged between the various mobile devices as well as the devices and the server. Processed information is sent over the network in the form of presence information to our web portal and can be shared with different applications. Based on the availability of different mobile devices used in SACAAR, the inference of complex activities is performed by the mobile device with higher resources in terms of battery and processing capabilities. The devices periodically check for the availability of other devices belonging to the user and his/her environment for such data exchange and processing.

The activity inference layer performs atomic and complex activity inference. The complex activity recognition (CAR) algorithm is used to recognize interleaved, concurrent and out of sequence activities. The algorithm also interacts with the repository which stores definitions of atomic and complex activities being inferred. The repository also stores associations between atomic activities and the various sensors that are used and is present inside the sensory layer. We work at two levels one is the atomic activity inference level and then use our CAR algorithm to infer complex activities. We infer four types of atomic activities namely body motion, object interaction, device activity and browser activity in our test-bed, but other atomic activities can similarly be added. We incorporate the use of other context information for atomic activities in addition to sensors directly linked to atomic activities for example accelerometer for body motion along with speed and direction of user. We use state of the art techniques for inference [4, 5] of atomic activities. Body motion such as sitting, standing, walking, etc are inferred using decision trees [4] which have been widely studied. We use a weighted voting sliding window mechanism to infer RFID object usage. A similar sliding window weighted voting mechanism is also applied to device and browser activity.

We collect spatial and temporal data in the sensory layer to create spatio-temporal situations. For example, user is in office at noon, in city at noon, or in home early morning. Activities always belong to some spatio-temporal situation and linking them can help in better understanding of activities being performed. These linkages can be created at run time and new activities can be added to situations if not linked previously. We initially create situations and link our pre-defined complex activities to these situations. The aim is to reduce the number of complex activities to look for during the inference process. We perform situation-aware reasoning based on the Context Spaces Model [6] to infer situations. The CAR algorithm is used to fuse together different sources of atomic activities and combine them to infer complex activities with the help of the situation and activity linkages provided by the spatio-temporal context complexity filter. The CAR algorithm is then used for inference of multiple complex activities which can be concurrent and interleaved. Thus, CAR algorithm works by taking atomic activities, context information and situations as input and outputs the complex activities. For the sake of brevity, we omit the algorithm details. Further implementation details on our test-bed can be found here [7]. We test and validate SACAAR using our test-bed in the social networking domain where we share activity based presence updates.

We used our SACAAR approach and inferred interleaved and concurrent complex activities using 2 subjects for 3 weeks. Subject one performed all activities while subject two performed only 13 activities out of the 16 . The subjects were given complete freedom to perform the previously defined complex activities in any interleaved and concurrent manner and in any order. It is important to note that our approach does not require training data to create activity models. It only uses domain knowledge to define complex activities and create links to atomic activities. Our CAR algorithm performed with an overall accuracy of $88.5 \%$ for interleaved, concurrent and out of sequence complex activities.

\section{CONCLUSION}

In this paper, we propose SACAAR architecture which infers atomic and complex activities by mapping them onto situations. We developed a novel system which uses multiple sensor and context information to infer atomic and complex activities that are interleaved and concurrent in nature. A testbed and prototype are built to validate SACAAR system. The experimental results show that activity inference with considerably high accuracy was achieved with minimal domain knowledge and without any training at complex activity level.

\section{REFERENCES}

[1] Yang, G.-Z., B. Lo, and S. Thiemjarus, Body Sensor Networks. 2006: Springer London.

[2] Kaptelinin, V., B. Nardi, and C. Macaulay, Methods \& tools: The activity checklist: a tool for representing the "space" of context. interactions, 1999. 6(4): p. 27-39.

[3] Adi, A. and O. Etzion, Amit - the situation manager. The VLDB Journal, 2004. 13(2): p. 177-203.

[4] Bao, L., Physical Activity Recognition from Acceleration Data under Semi-Naturalistic Conditions, in Department of Electrical Engineering and Computer Science. 2003, Massachusetts Institute of Technology.

[5] Stikic, M., et al. ADL recognition based on the combination of RFID and accelerometer sensing. in Pervasive Computing Technologies for Healthcare, 2008. PervasiveHealth 2008. Second International Conference on. 2008.

[6] Padovitz, A., S.W. Loke, and A. Zaslavsky, Towards a Theory of Context Spaces. Proceedings of the Second IEEE Annual Conference on Pervasive Computing and Communications Workshops, 2004.

[7] Saguna, S., A. Zaslavsky, and D. Chakraborty, CrysP: Multi-Faceted Activity-Infused Presence in Emerging Social Networks, in ruSMART 2010, Springer. 